\title{
Analyzing the Effect of Doping Concentration on Dielectric Properties of Polymeric Films
}

\author{
Renu Tyagi ${ }^{1, *}$, Yuvraj Singh Negi ${ }^{1}$, Millie Pant ${ }^{2}$ \\ ${ }^{1}$ Department of Polymer Science and Process Engineering, Saharanpur Campus, IIT Roorkee, India \\ ${ }^{2}$ Department of Applied Science and Engineering, Saharanpur Campus, IIT Roorkee, India \\ *Corresponding author: renutyagi80@gmail.com
}

Received March 27, 2014; Revised April 30, 2014; Accepted May 14, 2014

\begin{abstract}
In the present study two organic guest host system are being explored for electronic applications based on 2-methyl 4-nitroaniline (2-mNA)/polymethylmethacrylate (PMMA) and 2-mNA/ polyethersulfone (PES). Freestanding poled polymeric films have wide applications in nonlinear optics and in various photonics applications. Exploring dielectric capability of these films can be beneficial for many opto, electro and photonic applications. Low power loss is an essential characteristic for any electronic element and has been a challenging job for researchers and scientists. Forwarding in this direction, this paper focuses on capacitance of unpoled and poled polymeric films. Dissolving limit of guest material into polymer without showing any segration of guest material has been optimized for both host polymers. Capacitance is measured with different dose of 2-mNA by using 4294A Agilent Impedance Analyzer at room temperature. Here we are only manipulating the results that are optimized at $1 \mathrm{~K} \mathrm{~Hz}$ frequency. It is observed that capacitance increases with increasing 2-mNA content into polymer but PMMA/2-mNA system shows more dielectric strength than PES/2-mNA system. The impact of half an hour contact poling by using $6 \mathrm{KV} / \mathrm{cm} \mathrm{DC}$ electric field at $120^{\circ} \mathrm{C}$ temperature on dielectric capability is also optimized. It is observed that how; to be poled the guest host films can affect the dielectric strength of both polymeric systems. Without describing electric power consumption in terms of power loss, electrical quality of any component is incomplete. Thatswhy dissipation factor have been evaluated for both guest host system at $1 \mathrm{~K}$ Hz frequency. Dissipation factor rises with the 2-mNA doping concentration but after poled the films, it reduces drastically. PES/2-mNA system showed low power loss than PMMA/2-mNA system. The structural characterizations of all films are carried out by XRD, which shows an amorphous nature of PES/2-mNA and PMMA/2-mNA film samples. Absorbance measured by UV-Vis Spectroscopy reveals that undoped film samples has high transmittance in the UV-Vis region and doped film samples shows absorption band up to $450 \mathrm{~nm}$. The films are characterized by Scanning Electron Microscopy and FT-IR Spectroscopy for bulk properties evaluation.
\end{abstract}

Keywords: 2-mNA, contact poling, organic polymers, guest host system, capacitance, dissipation factor

Cite This Article: Renu Tyagi, Yuvraj Singh Negi, and Millie Pant, "Analyzing the Effect of Doping Concentration on Dielectric Properties of Polymeric Films." Journal of Materials Physics and Chemistry, vol. 2, no. 2 (2014): 15-19. doi: 10.12691/jmpc-2-2-1.

\section{Introduction}

Polymer material is widely being used in various devices as insulating material and for optoelectronic applications $[1,2]$. This is due to their unique properties such as light weight, high flexibility, and ability to be fabricated at low temperature and low cost. There are several organic dielectric materials that have been studied for optical, electrical and photonic applications [3,4]. Some of these are polyvinyl phenol, polyimide, polymethylmethacrylate, poly carbonate, polystyrene, polyethersulfone etc. [5,6,7]. Poled polymers also have been proposed for use in a number of electro-optics applications, such as telecommunications switching devices, sensors, modulators, actuators and data storage devices [8]. During the poling process, the molecules of chromophore in a guest-host polymer system spontaneously align under a strong applied electric field. The poling process is usually carried out near the glass transition temperature ( $\mathrm{Tg}$ ) of the polymer host, i.e., the point at which the polymer host softens, allowing chromophore rotation and alignment to occur. However, when cooled below $\mathrm{T} g$ of the polymer host the aligned chromophores become locked in their new orientation within the polymer host [9]. When poled in this way, guest-host polymers can exhibit many useful electro-optic properties [10]. During poling process, at molecular level the chromophores must exhibit excited state close in energy to the ground state with large dipole moment difference between these states. To fulfill these requirements, the chromophores must possess electron donating group and electron withdrawing group connected by highly conjugated pi electronic system [11]. After that these chromophores are incorporated in to polymer backbone either covalently or by guest host system. The simplicity in preparation of non-covalently 
bonded guest host materials might be an attraction if suitable stability of polar alignment can be attained $[12,13]$.

Polymethyl methacrylate (PMMA) [14,15,16] and polyethersulfone (PES) [9] also has attracted high attention for electrical and optical applications. These organic polymers are chosen to be dielectric materials in organic thin film transistor and organic field effect transistor because it has good insulation properties such as low dielectric constant and low dielectric loss over a wide range of frequency. In this work, PMMA and PES are used as host material. We prepared guest host polymer thin films with different concentration of 2-methyl 4nitroaniline (2-mNA).

Our aim is to investigate the effect of chromophoric material concentration on the dielectric properties of polymeric thin film. In order to make proper analysis, chromophoric material is incorporated in to polymeric material up to $18 \%$ of weight \% of polymer with step size of 4 . To make proper alignment of 2-mNA in to polymeric film, the films are poled at $120{ }^{\circ} \mathrm{C}$ temperatures under external DC electric field of $6 \mathrm{KV} / \mathrm{cm}$ strength. After poling for half an hour, heating are switched off and films are cooled down to room temperature but electric field is applied continuously till the films cool down. The poled and unpoled films are characterized for dielectric strength and power loss. The properties analyzed in this paper are (i) capacitance $(\mathrm{pF})$ and (ii) dissipation factor (mU). Capacitance and dissipation factor is observed with different concentration of 2-mNA for both polymeric thin films (PMMA-2mNA and PES-2mNA). It is observed that molecular alignments of chromophoric material (2-mNA) minimize the dissipation factor of prepared films significantly.

\section{Materials and Methods}

In this report organic material 2-mNA of optical grade (Merck) is used as a chromophore molecule, and organic polymeric material PMMA (Merck) and PES (Solvay Chemicals) are used as host polymer.

Tough, transparent and flexible films of PMMA/PES doped with appropriate amount of 2-mNA are prepared by solution cast method in dimethyl sulphoxide solvent. Total solid weights are kept $1 \mathrm{gm}$ to make all films. It is

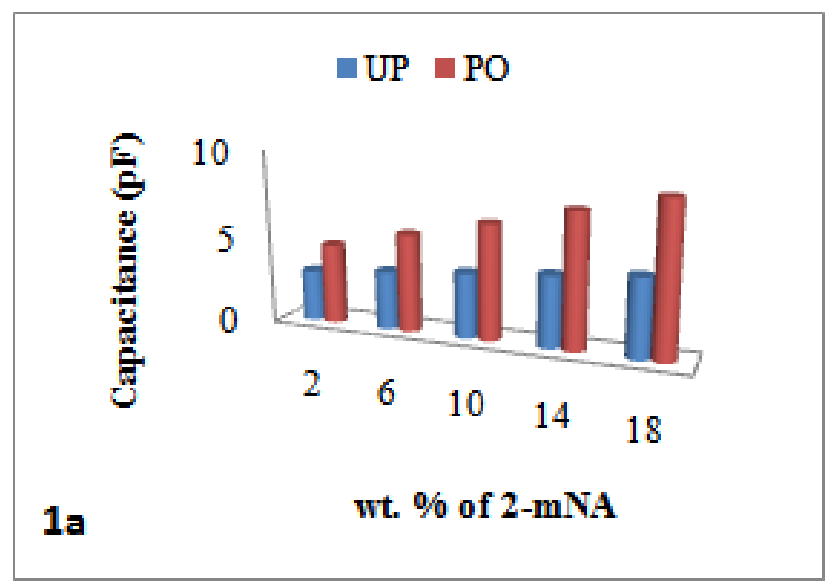

observed that PMMA and PES can incorporate 18 weight \% of 2-mNA in polymer matrix without showing any visible aggregate of 2-mNA.

Therefore, 2-mNA is added in the range of 2 to $18 \%$ by weight $\%$ of host polymer taking step size of 4 . The films were dried in vacuum oven for $4-5 \mathrm{~h}$ at $100 \mathrm{C}$ to remove all residual solvent. After drying, colored transparent films are obtained and subjected to electrical characterization. The films prepared by using PMMA and 2-mNA are denoted by PM system and the films prepared by using PES and 2-mNA are denoted by PS system.

The doped polymer films are poled by contact poling method at $120^{\circ} \mathrm{C}$ temperature with an applied dc electric field $(6 \mathrm{KV} / \mathrm{cm})$. After being poled for an half h, the film samples are cooled to room temperature maintaining the poling electric field unchanged. Then the 2-mNA chromophore molecules in the polymer host are aligned along the direction of the electric field and locked in by cooling. Unpoled films are denoted by UP and poled films are denoted by PO. The electrical characterization of unpoled and poled film samples have been done.

Agilent Impedance Analyzer 4294A is used to determine the capacitance and dissipation factor of unpoled and poled polymer films at room temperature. The behavior of capacitance and dissipation factor with different dose of 2-mNA are studied. The effect of chromophore molecule alignment on the above said properties are evaluated. The results are exemplified at a particular frequency of $1 \mathrm{~K} \mathrm{~Hz}$.

\section{Results and Discussion}

The main aim of our study is to observe the electric property of organic materials viz PMMA/PES and 2-mNA as host-guest system. The effect of poling field strength on the electric property is an interesting subject of research. In our study the focus is on the dielectric capability of both materials along with doping of chromophore molecules in different percentage. Because minimum loss of power is a necessary requirement for any electronic component, the second emphasis is on the dissipation factor. Interesting results are found for both guest host system. Detailed explanation of electric properties for both system (PM and PS) is given below.

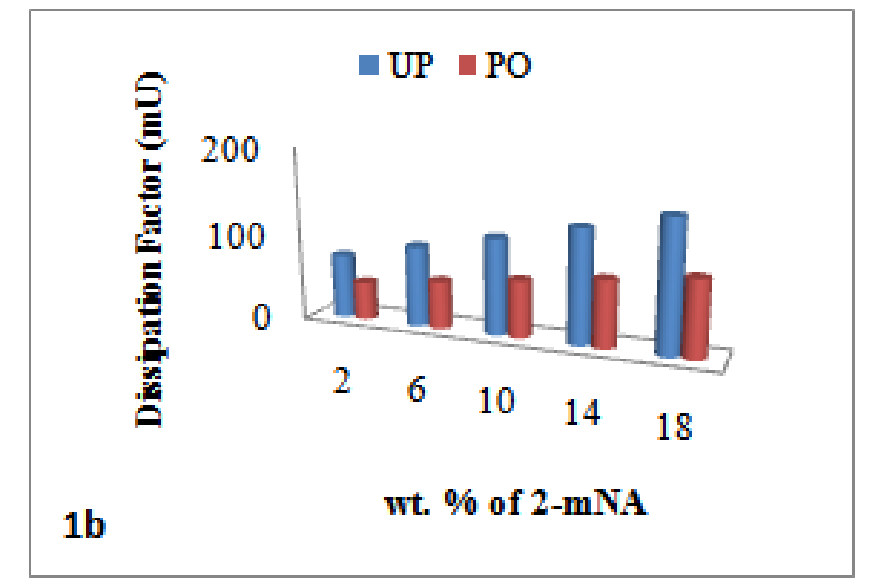

Figure 1. Relationship of (a) capacitance (b) dissipation factor with 2-mNA concentration for PMMA/2-mNA system 


\subsection{PM System (PMMA/2-mNA)}

The capacitance for unpoled and poled film samples with 2-mNA concentration have been shown in Figure 1. All UP (unpoled) and PO (poled) film samples are characterized at $1 \mathrm{~K} \mathrm{~Hz}$ frequency, at room temperature for capacitance. The graph precise that as dose of 2-mNA content rises in polymeric films, the capacitance of all film samples (UP and PO) enhances respectively. In UP film samples the 2-mNA molecules are randomly oriented. After poling these film samples for half an hour at $120^{\circ} \mathrm{C}$

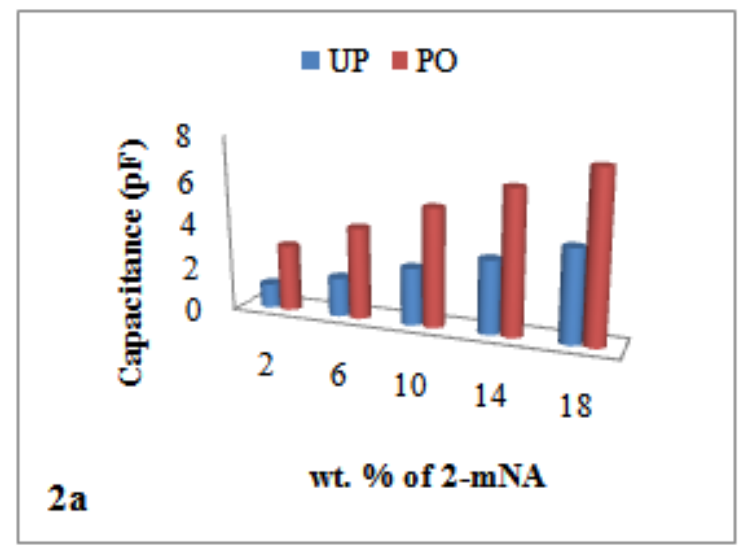

temperature, the molecules of 2-mNA becomes aligned in the electric field direction. Due to the alignment of 2mNA molecules the PO film samples shows higher value of capacitance. Also, the enhancement in capacitance is of significant range.

The losing power quality of UP and PO film samples are shown in Figure 2. From this Figure, it is clearly seen that, the presence of 2-mNA molecules in polymeric film results in electric power loss. But when these molecules have been locked in electric field direction, the consumption of electric power can be minimized very well.

Figure 2. Relationship of (a) capacitance (b) dissipation factor with 2-mNA concentration for PES/2-mNA system

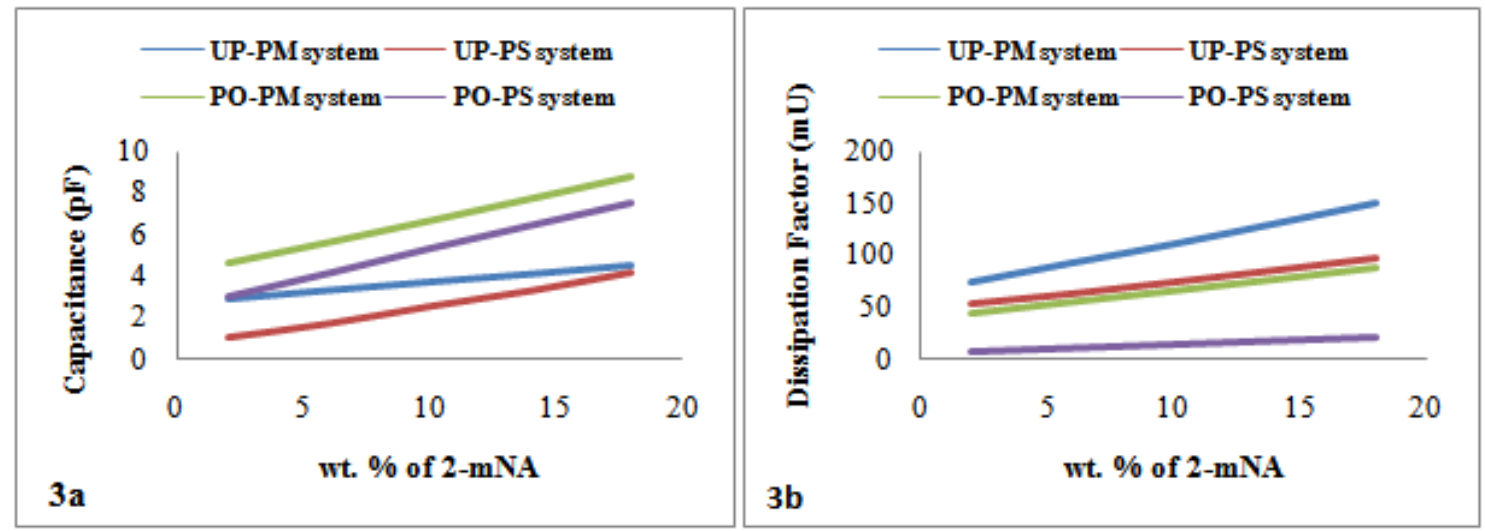

Figure 3. Difference of observed (a) capacitance (b) dissipation factor for both system
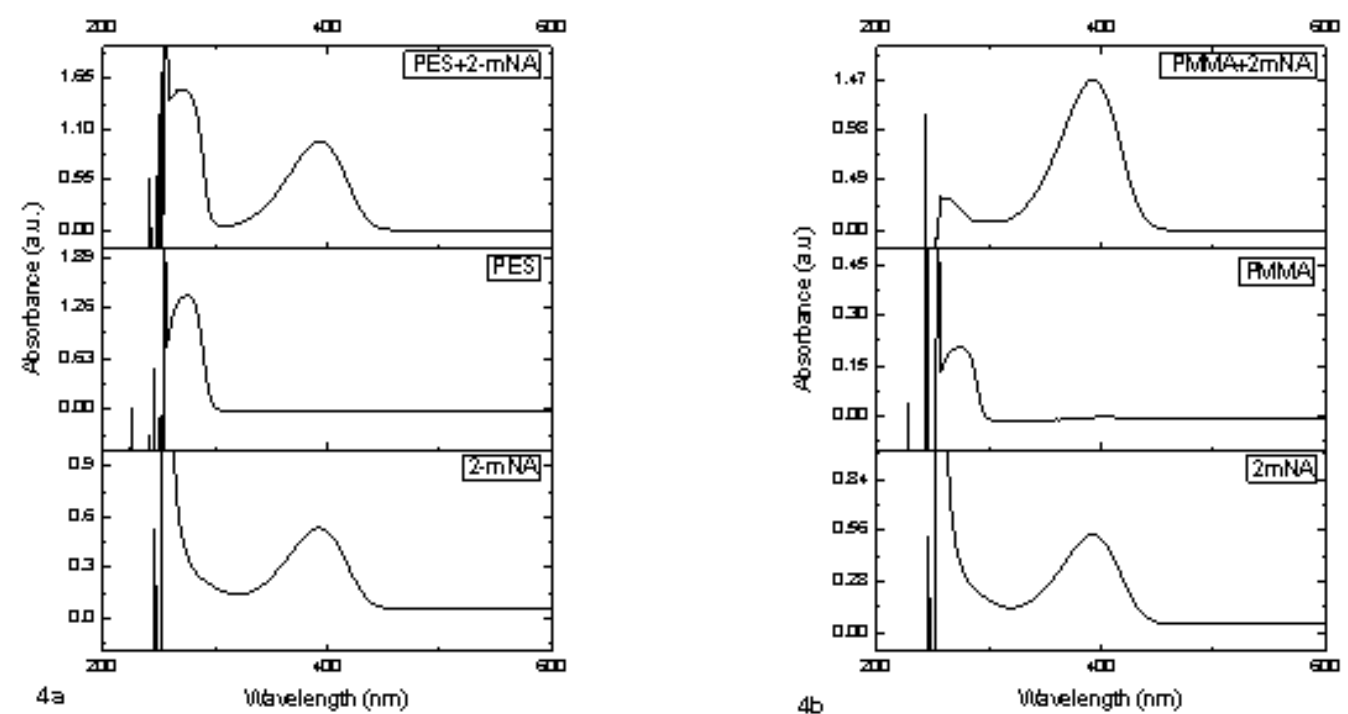

Figure 4. UV-Vis Spectra of (a) PES/2mNA films and (b) PMMA/2mNA films 


\subsection{PS System (PES/2-mNA)}

The electronic property of PS film samples (UP and PO) have been shown in Figure 3 and Figure 4. Films become more dielectric with dose of 2-mNA. From the point of electrical quality, the alignment of guest molecules is beneficial for PS film samples.

\subsection{PM System and PS System}

It is meaningful to compare the quality of different materials. In this report, efforts have been done to visualize the electric quality of PMMA and PES with composition of 2-mNA. PMMA has been seen to be high dielectric than PES for both UP and PO system (Figure 5).

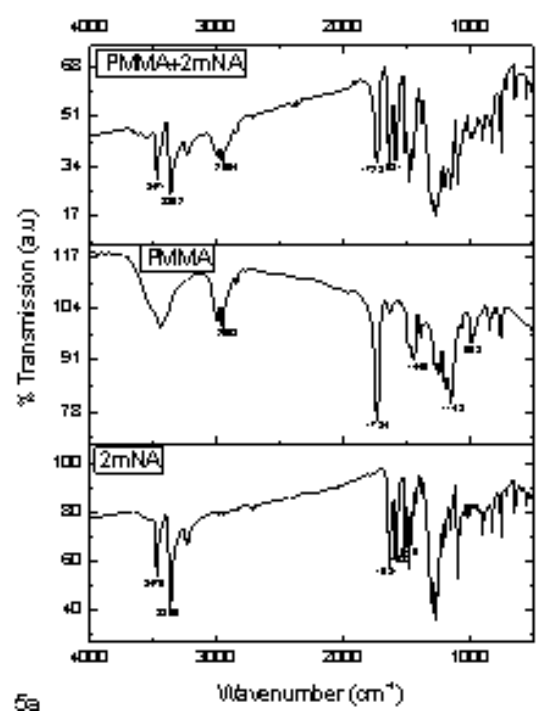

In terms of electric power loss, PES has been proved to be a better material than PMMA (Figure 6).

\subsection{Ultra-Violet Studies}

SHIMADZU UV-1800 spectrophotometer used to determine the UV-Vis light absorption of the controlled and doped PES and PMMA films in the range 200 to 800 nm (Figure 4a \& Figure 4b). PMMA and PES exhibits higher transmission through the visible wavelength range. Very little UV absorption untill $300 \mathrm{~nm}$ but in the case of 2-mNA doped films, the absorption band is seen in the range of 310-450 which is due to high energy absorption by 2-mNA guest molecules.

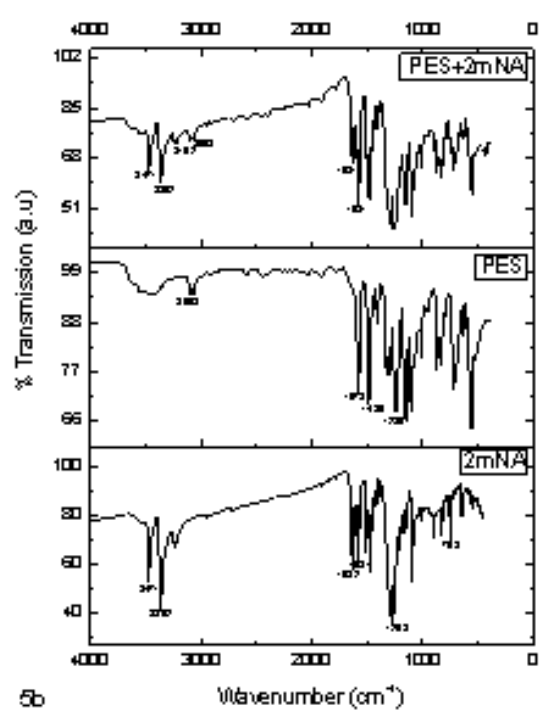

Figure 5. FTIR Spectra of (a) PMMA/2mNA and (b) PES/2mNA films

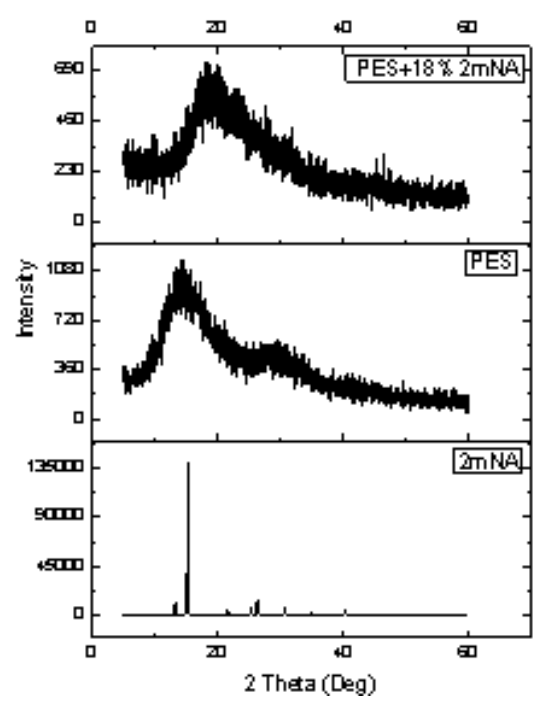

Figure 6. XRD Spectra of (a) PES/2mNA and (b) PMMA/2mNA films

\subsection{FTIR Studies}

The type of interaction between the guest and host molecules recorded by PERKIN Elmer FTIR Spectrophotometer, and the characteristics vibration modes are provided (Figure 5a \& Figure 5b). IR spectral bands for PMMA obtained at 1148, 1449, 1734 and 2953 $\mathrm{cm}^{-1}$ are due to ether band, $\mathrm{C}-\mathrm{H}$ bending of $\mathrm{CH} 2$ group, carbonyl group and $\mathrm{C}-\mathrm{H}$ stretching respectively. FTIR peaks of 2-mNA are obtained at 3360 and $3470 \mathrm{~cm}^{-1}$ corresponding to the symmetric and asymmetric modes of free $\mathrm{NH}_{2}$ groups. Aromatic bands at 1578 and $1486 \mathrm{~cm}^{-1}$ are characteristic for PES. The presence of 2-mNA confirmed with the peaks of $>\mathrm{NH}$ stretching vibrations at $3102,3063 \mathrm{~cm}^{-1}$ and $>\mathrm{NH}$ bending vibration at $1583 \mathrm{~cm}^{-1}$ additionally in the doped system. $\mathrm{NH}$ bending modes at $1630 \mathrm{~cm}^{-1}, \mathrm{No}_{2}$ stretching mode at $1519 \mathrm{~cm}^{-1}$ confirms the presence of 2-mNA. 


\subsection{XRD Studies}

The XRD characterization confirms the amorphous nature of the PES and PMMA systems for both doped and undoped films (Figure 6a \& Figure 6b). There are no phase aggregations observed even up to $18 \%$ 2-mNA doping. The 2-mNA crystals did not affect the amorphous nature of host PES and PMMA.

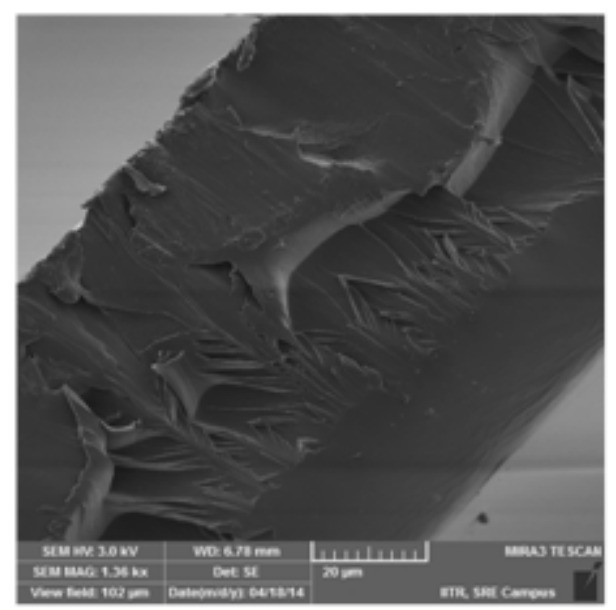

Figure 7. SEM Micrograph of doped PES films with 18\% 2-mNA

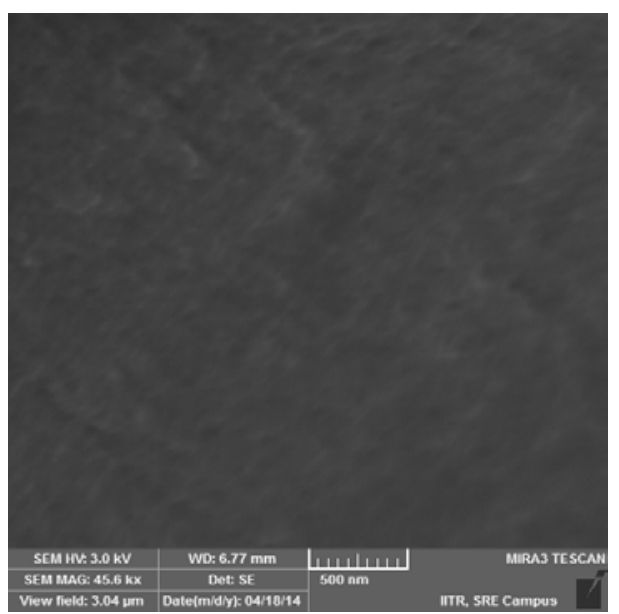

Figure 8. SEM micrograph of doped PMMA films with 18\% 2mNA

\section{Conclusions}

We introduced the polymeric thin films which are based on guest host system containing PMMA and PES as host material and 2-mNA as guest material. Guest material was dissolved up to limit until transparency of films is maintained. In our study, the dissolving range of guest polymer into host polymers is optimized as 18 weight \% of host polymers. Prepared films have significant insulator quality and it could be improved with 2-mNA percentage respectively. Good enhancement in capacitance is observed by doing half an hour poling under $6 \mathrm{KV} / \mathrm{cm}$ electric field strength. PMMA-2mNA systems are more dielectric as compare to PES-2mNA system. Dissipation factor is also optimized for both systems. PES-2mNA system have low tendency to waste the electric power than PMMA-2mNA system. Alignment of 2-mNA molecules is proved a successful action to control the power loss for the films made by both guest host system. We will discuss the

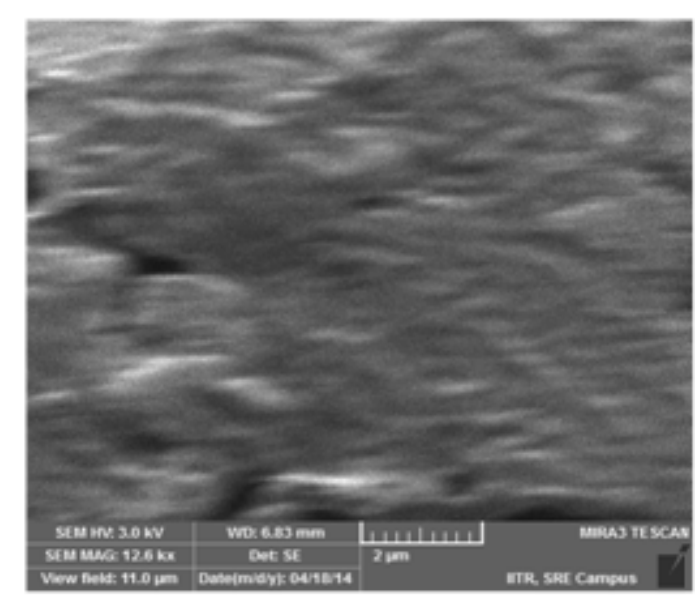

\subsection{Scanning Electron Microscopy}

The characteristics features of films obtained from Scanning Electron Microscopy are shown in Figure 7Figure 8. There is no aggregate formation is observed even up to18\% 2-mNA doping. Figure confirms the overall and orderly distribution of 2-mNA molecules into the films.

behaviors of capacitance over wide frequency range in our next report.

\section{Acknowledgement}

First author, Renu Tyagi, thanks the Institute Instrumentation centre, IIT Roorkee for supporting the research work for Organic Polymeric Materials for Device Applications.

\section{References}

[1] S-W Jung et al J. Appl. Phys. 108 102810(1) (2010).

[2] Y Lina, J Zhang, L Brzozowski, E H Sargent and E Kumacheva J. Appl. Phys. 91(1) 522 (2002).

[3] A K Arof, M Z Kufian, M F Syukur, M F Aziz, A E Abdelrahman and S R Majid Electrochimica Acta 7439 (2012).

[4] S Gunasekaran, K S Kannan, S Loganathan, G Anand and S Kumaresan Indian. J. Phys. 87(12) 1189 (2013).

[5] Ji H Park, D K Hwang, J Lee, S Im and E Kim Thin Solid Films 5154041 (2007).

[6] H A Bioki and M B Zarandi Indian J. Phys. 86(6) 439 (2012).

[7] B H Rudramadevi, K Thilagavathi and S Buddhudu Indian J. Phys. 86(11) 997 (2012).

[8] H S Nalwa, T Watanabe, K Ogino, H Sato and S Miyata J. Mater. Sci. 333699 (1998)

[9] R K Goyal, S R Damkale, U P Mulik, Y S Negi, J W Dadge and R C Aiyer Adv. Mat. Lett. 1(3) 264 (2010).

[10] R C Hoffman et al Opt. Mater. Exp. 1(1) 67 (2011).

[11] B M Boaz, R S Selvaraj, K S Kumar and S J Das Indian J. Phys. 83(12) 1647 (2009).

[12] A Kozanecka-Szmigiel, K Świtkowski, B Pura, E SchabBalcerzak and M Siwy Photo. Lett. POLAND 1(4) 181 (2009).

[13] W Shi, C Fang, Q Pan, X Sun, Q Gu, D Xu J. Mater. Sci. Lett. 19 147 (2000).

[14] S A Hashmi, A Kumar and S K Tripathi J. Phys. D: Appl. Phys. 406527 (2007).

[15] N Kalra, D K Chaturvedi, S Kumar and S Arora Indian J. Pure \& Appl. Phys. 45618 (2007).

[16] A Tiwari, A K Gupta, R Bajpai and J M Keller Indian J. Phys. 85(11) 1581 (2011). 